\title{
FERRAMENTA DE AUXÍLIO NO PROCESSO DE MEDIÇÃO DE ENERGIA ELÉTRICA UTILIZANDO INTELIGÊNCIA COMPUTACIONAL
}

\author{
Marco Antônio Silva Pereira ${ }^{1}$
}

\section{RESUMO}

Este artigo aborda assuntos relacionados aos problemas encontrados na medição de energia elétrica em decorrência de distorções harmônicas da rede. Tais distorções podem ser capazes de provocar erros consideráveis, acarretando, consequentemente, em cobrança de uma quantidade indevida de energia pela empresa fornecedora. Tendo em vista esse problema, o objetivo deste artigo consiste em tornar o processo de medição de energia elétrica mais eficiente por meio de conceitos referentes a Redes Neurais Artificias e Redes Neuro-Fuzzy.

Palavras-chave: Medição de energia elétrica. Distorções Harmônicas. Redes Neurais Artificiais. Redes Neuro-Fuzzy.

\section{A TOOL TO ASSIST IN THE PROCESS OF ELECTRIC ENERGY MEASUREMENT BY THE USE OF COMPUTATIONAL INTELLIGENCE}

\begin{abstract}
This article discusses issues related to problems encountered on electrical energy measurement due to harmonics distortions in the network. Such distortions may be able to cause considerable errors and consequently an undue amount of energy collection by the supplier. In view of this problem, the objective of this article is to make the process of measuring more efficient electricity through concepts related to Artificial Neural Networks and Neuro-Fuzzy Networks.
\end{abstract}

Keywords: Electrical energy measurement. Harmonic Distortions. Artificial Neural Networks. Neuro-Fuzzy Networks.

\section{INTRODUÇÃO}

O sistema elétrico brasileiro, a partir dos anos 80 , começou a sofrer grandes mudanças em função dos avanços tecnológicos proporcionados pelo crescimento da utilização da eletrônica de potência. Surgiu, então, uma preocupação referente à qualidade da energia elétrica, tanto do ponto de vista dos fornecedores quanto dos consumidores, devido aos distúrbios causados pelas características dos novos dispositivos conectados na rede elétrica.

\footnotetext{
${ }^{1}$ Mestrando em Engenharia Elétrica pela Universidade Federal de São João Del Rei (UFSJ), graduado em Engenharia Elétrica pelo Instituto Federal de Minas Gerais - Campus Formiga e atualmente é professor da educação básica no IFMG - Campus Formiga. E-mail: marcoengeletrica.ifmg@hotmail.com.

ForSci.: r. cient. IFMG campus Formiga, Formiga, v. 3, n. 1, p. 31-41, jan./jun. 2015.
} 
Pode-se dizer, ainda, que a utilização dessas novas tecnologias obrigou os consumidores e fornecedores a buscarem uma energia sem desvios na tensão, corrente ou frequência que resultem na falha ou má operação dos equipamentos dos consumidores (DUGAN; MCGRANAGHAN; BEATY, 2012).

Dentre os distúrbios, que podem ser relacionados com a falha ou operação indevida de equipamentos conectados na rede elétrica, destacam-se as distorções harmônicas. Tais distorções estão relacionadas com as formas de onda da corrente ou da tensão e são capazes de produzir comportamentos indevidos nos medidores convencionais de energia elétrica (RODRIGUES, 2009).

O medidor de energia convencional, denominado como medidor de indução, baseia-se no fenômeno de interação eletromagnética. Esse tipo de medidor é amplamente utilizado devido à sua simplicidade, robustez, exatidão e desempenho ao longo dos anos. Porém, quando são submetidos a tensões e a correntes distorcidas, tais medidores apresentam erros de medição (SOUZA et al., 2010).

A medição da energia elétrica é empregada pela concessionária (fornecedora de energia elétrica) para possibilitar o faturamento adequado da quantidade de energia elétrica ativa consumida por cada unidade consumidora, dentro de uma determinada tarifa estabelecida (SILVA, 2008). Portanto, surge a necessidade de adotar técnicas para minimizar os erros de medição de energia para que a concessionária e os consumidores não sejam prejudicados com tarifações inadequadas.

Neste contexto, propõe-se o uso de ferramentas, baseadas em inteligência computacional, para auxiliar o processo de medição de energia elétrica de modo com que esse apresente resultados apropriados, mesmo em ambientes repletos de conteúdo harmônico. Especificadamente, foram adotadas técnicas de Redes Neurais Artificiais (RNAs) e de Redes Neuro-Fuzzy.

\section{REFERENCIAL TEÓRICO}

\subsection{Distorções Harmônicas}

Para que se possa compreender o significado das distorções harmônicas deve-se, primeiramente, considerar alguns conceitos sobre a Teoria de Fourier. De acordo com essa teoria, qualquer sinal periódico pode ser representado pela soma de diversos sinais senoidais. Existe, nesse caso, a superposição de uma componente senoidal fundamental e outras 
senóides, denominadas como harmônicas, que apresentam frequências múltiplas inteiras da frequência da componente fundamental (SOARES, 2004).

A Figura 1 mostra a representação de uma onda dente de serra e algumas de suas respectivas componentes harmônicas.

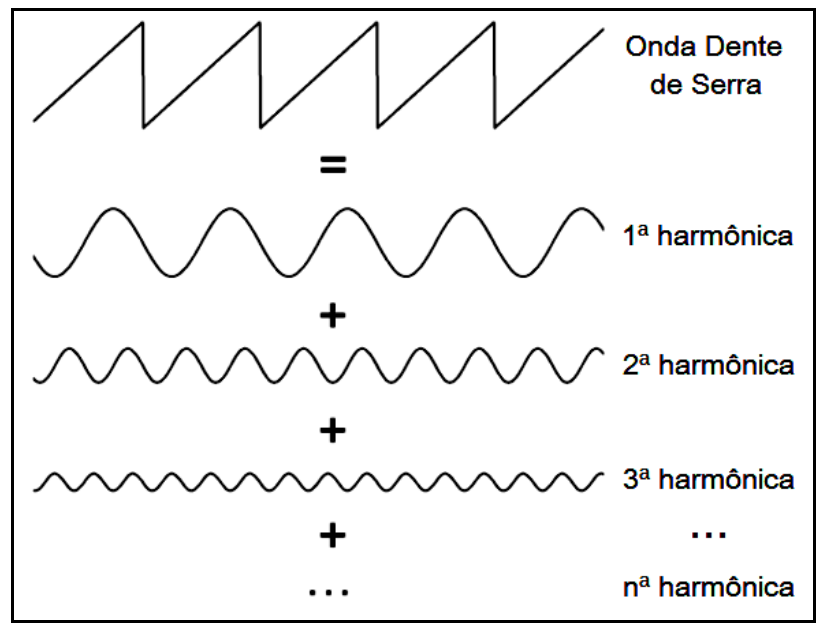

Figura 1- Representação de uma onda dente de serra e suas respectivas componentes harmônicas Fonte: Soares (2004).

Pode-se dizer que, se a frequência da onda dente de serra é $1 \mathrm{~Hz}$, a primeira componente senoidal tem $1 \mathrm{~Hz}$ e pode ser chamada de primeira harmônica ou componente fundamental. As demais senóides possuem frequências iguais a múltiplos inteiros da frequência da componente fundamental e suas respectivas amplitudes são diferentes. Considerando-se a forma de onda senoidal da rede elétrica, as harmônicas com maior amplitude de sinal são aquelas cujas frequências são múltiplas ímpares da frequência fundamental (SOARES; ANDRADE, 2004).

As distorções harmônicas da rede elétrica são, portanto, resultado de tensões ou correntes senoidais com frequências múltiplas inteiras da frequência que o sistema de suprimento é projetado a operar, $60 \mathrm{~Hz}$ no caso do sistema de potência brasileiro. A origem dessas distorções é proveniente de características não lineares de dispositivos e cargas conectados no sistema de energia (DUGAN; MCGRANAGHAN; BEATY, 2012).

Para quantificar a intensidade das distorções harmônicas deve-se descrever o espectro harmônico completo do sinal com magnitudes e ângulos de fase de cada componente harmônico. O indicador mais utilizado para essa quantificação é o Distorção Harmônica Total (DHT) que pode estar relacionado a distorções de tensão ou de corrente e é definido como a porcentagem da amplitude das distorções em relação à componente fundamental (DUGAN; 
MCGRANAGHAN; BEATY, 2012). A Equação 01 é uma expressão referente ao cálculo da Distorção Harmônica Total da Tensão $\left(D H T_{V}\right)$ e a Equação 02 ao cálculo da Distorção Harmônica Total da Corrente $\left(D H T_{F}\right)$.

$$
\begin{gathered}
D H T_{V}=\frac{\sqrt{\left(\sum_{n=2}^{\infty} U_{n}^{2}\right)}}{U_{-}} \\
D H T_{F}=\frac{\sqrt{\left(\sum_{n=2}^{\infty} I_{n}^{2}\right)}}{I_{1}}
\end{gathered}
$$

Onde:

$U_{1}$ - é o valor da tensão fundamental;

$U_{n}$ - é o valor da tensão de ordem harmônica n;

$I_{1}$ - é o valor da corrente fundamental;

$I_{n}$ - é o valor da corrente de ordem harmônica $\mathrm{n}$.

\subsection{Inteligência Computacional}

Inteligência computacional é a área da ciência que estuda a teoria e a aplicação de técnicas inspiradas na natureza, tais como Redes Neurais Artificiais e Lógica Fuzzy. Tais técnicas podem ser utilizadas para detectar os erros contidos em medições de energia elétrica, em decorrência de distorções harmônicas, e fornecer estimativas mais adequadas para a quantidade de energia consumida por cada usuário da rede elétrica.

\subsubsection{Redes Neurais Artificiais}

As redes neurais artificiais (RNAs) são modelos computacionais baseados no funcionamento do sistema nervoso dos seres vivos. Tais modelos possuem a capacidade de aquisição e manutenção do conhecimento por meio de um conjunto de unidades de processamento, caracterizadas por neurônios artificiais, dispostas em uma ou mais camadas interligadas por um grande número de conexões. $\mathrm{Na}$ maioria dos modelos essas conexões estão associadas a pesos com a função de armazenar o conhecimento, representado no modelo, e servir para ponderar a entrada recebida por cada neurônio da rede (SILVA; SPATTI; FLAUZINO, 2010). 
De modo geral, uma RNA é caracterizada pela topologia de sua arquitetura. Essa topologia está relacionada com as interligações entre os neurônios da rede, pelo algoritmo de treinamento, que ajusta os pesos e conexões dos neurônios, e pela função de ativação dos neurônios, responsável pelo processamento do conjunto de informações recebidas e pela saída do neurônio. Desse modo, a escolha da rede que melhor se adapta a cada problema, em específico, pode ser determinada a partir de testes com várias topologias diferentes (ELLER, 2003 apud SOUZA et al., 2010).

\subsubsection{Lógica Fuzzy}

A Lógica Fuzzy, também chamada de Lógica Nebulosa, tem por objetivo modelar, de forma aproximada, o raciocínio dos seres humanos, visando ao desenvolvimento de sistemas computacionais capazes de tomar decisões racionais em um ambiente de incertezas e imprecisões. Tal lógica utiliza a ideia de que todas as coisas (temperatura, altura, velocidade, etc.) admitem graus de pertinências. Dessa forma, a lógica Fuzzy pode ser considerada como um conjunto de princípios matemáticos para a representação do conhecimento baseado no grau de pertinência, ou grau de verdade, de termos relacionados a diferentes classes.

É fato que, para muitos problemas reais, a imprecisão dos dados e a incerteza do conhecimento são, por natureza, parte do problema em si, e raciocinar considerando esses aspectos sem uma fundamentação adequada pode gerar inferências imprecisas. A Lógica Fuzzy, por sua vez, coloca-se como um instrumento robusto para uma representação mais adequada do conhecimento devido à sua capacidade de lidar com incertezas, raciocínio aproximado, etc. (MARRO et al., 2010).

\subsubsection{Redes Neuro-Fuzzy}

Apesar de apresentar muitas vantagens, a Lógica Fuzzy possui algumas limitações que incentivaram o desenvolvimento de novas metodologias que consistem de uma combinação de sistemas Fuzzy e outras técnicas. Esses sistemas, denominados como híbridos, foram propostos com o intuito de prover adaptabilidade aos modelos que utilizavam apenas a abordagem Fuzzy. Um exemplo de sistemas híbridos é a rede Neuro-Fuzzy adaptativa (Adaptive Neuro-Fuzzy Inference System - ANFIS). Tal rede consiste de uma rede neural cuja ideia básica é implementar um sistema de inferência Fuzzy por meio de uma arquitetura 
paralela distribuída. Desse modo, os parâmetros associados com as funções de pertinência da Lógica Fuzzy são ajustados por meio de um algoritmo de aprendizado.

A escolha da topologia Neuro-Fuzzy mais eficiente para resolver cada problema, assim como é feito para os modelos de RNA, pode ser obtida por meio de testes. Nesse caso, variam-se as quantidades e tipos de funções de pertinência utilizados no sistema de inferência. Dentre as características do sistema ANFIS, pode-se destacar a sua utilização em problemas de aproximação de função e identificação de sistemas (TEIXEIRA; CORRÊA; ASSIS, 2007).

\section{METODOLOGIA}

Para realizar testes de algumas metodologias de mapeamento dos erros de medição de energia elétrica, utilizou-se como base de dados e de resultados um sistema que está disponível no artigo da referência (SOUZA et al., 2010). Nessa referência, foram realizados 24 ensaios para gerar um banco de dados. Tais ensaios consistiram na comparação dos valores medidos por um medidor padrão e por um medidor com classe de precisão de $0,5 \%$ e, para verificar os efeitos das distorções harmônicas, inseriu-se no circuito de medição um gerador de formas de onda distorcidas.

A metodologia adotada baseou-se, de forma geral, na implementação de várias arquiteturas de redes neurais, alterando-se o número de neurônios das camadas intermediárias das RNAs e o número de épocas de treinamento a ser executado. Para determinar a arquitetura mais eficiente, levaram-se em consideração os erros entre as saídas desejadas (valores reais de medição) e as obtidas na simulação RNA de cada arquitetura.

De modo similar, este artigo propõe várias outras arquiteturas de redes com o intuito de encontrar um método mais eficiente que a rede obtida pelo artigo base. Utilizou-se o mesmo banco de dados, assim como os ensaios especificados pelo artigo, para estabelecer critérios efetivos de comparação. Além de Redes Neurais Artificiais, testaram-se topologias de redes Neuro-Fuzzy para mapear o processo de medição descrito.

Para realizar as simulações referentes às RNAs propostas, utilizou-se a Neural Network Toolbox, do software Matlab. O processo de treinamento executado considerou, como regra de aprendizagem, o algoritmo do Gradiente Conjugado Dimensionado ("trainscg”) e a função de ativação Tangente Hiperbólica (“tansig”) para todos os neurônios. Para realizar as simulações referentes às redes Neuro-Fuzzy propostas, utilizou-se a Adaptive Neuro-Fuzzy Modeling Toolbox, do software Matlab. Nesse caso, as redes foram obtidas por meio da alteração do número de épocas e do número de funções de pertinência de cada 
entrada da rede e considerou-se a função Gaussiana ("gaussmf') para todas as funções de pertinência e, para treinamento da rede, um algoritmo híbrido.

Todas as simulações propostas foram executadas, valendo-se dos 20 ensaios utilizados pelo artigo base para realizar o treinamento das redes e, os 4 ensaios restantes, foram utilizados para realizar a validação das mesmas.

\section{RESULTADOS E DISCUSSÕES}

Os valores de tensão, corrente, $D H T_{V}$ e $D H T_{I}$ foram considerados como entradas para as redes especificadas e os valores resultantes do medidor padrão forneceram estimativas para as saídas desejadas. Desse modo, a Tabela 1 descreve resultados obtidos para diferentes RNAs.

Tabela 1- Resultados para RNAs similares com diferentes épocas de treinamento

\begin{tabular}{ccc}
\hline Neurônios & $\mathrm{N}^{\mathrm{o}}$ de Épocas & Erro Médio (\%) \\
\hline \multirow{2}{*}{7} & 1000 & 1,13 \\
& 2000 & 1,17 \\
& 3000 & 1,09 \\
\hline \multirow{2}{*}{9} & 1000 & 1,01 \\
& 2000 & 1,06 \\
& 3000 & 0,72 \\
\hline \multirow{2}{*}{10} & 1000 & 1,02 \\
& 2000 & 1,22 \\
& 3000 & 1,11 \\
\hline \multirow{2}{*}{12} & 1000 & 1,17 \\
& 2000 & 1,15 \\
\hline
\end{tabular}

Fonte: Do autor, 2014.

A Tabela 1 aborda redes com as mesmas quantidades de neurônios propostas pelo artigo base. Nesse caso, variou-se apenas o número de épocas de treinamento obtendo, para o caso da RNA com 9 neurônios na camada intermediária e número de épocas de treinamento igual a 3000, um erro relativo médio (para o treinamento) de $0,72 \%$ que é um resultado melhor do que o obtido pela rede com melhor desempenho encontrado pelo artigo base. A Tabela 2 mostra resultados para outras topologias de RNA e a Tabela 3 mostra resultados obtidos por meio da simulação de redes Neuro-Fuzzy, com a variação do número de funções de pertinência e quantidade de épocas de treinamento. 
Para as topologias com número de pertinências maior que 3 , necessitou-se de um tempo elevado de processamento em relação às demais topologias. Como a topologia que utilizou 3 funções de pertinência atingiu resultados satisfatórios, considerando o valor de Erro médio e, também, o tempo de simulação, realizaram-se, para esse caso, novas simulações, aumentando o número de épocas de treinamento. Os resultados obtidos podem ser verificados pela Tabela 4.

Tabela 2 - Resultados para RNAs com topologias diferentes

\begin{tabular}{ccccc}
\hline Neurônios & $\mathrm{N}^{\text {o }}$ de Épocas & Erro Médio (\%) & $\mathrm{N}^{\circ}$ de Épocas & Erro Médio (\%) \\
\hline \multirow{2}{*}{15} & 100 & 2,95 & 1000 & 1,23 \\
& 200 & 2,22 & 2000 & 1,17 \\
& 300 & 1,23 & 3000 & 0,84 \\
\hline \multirow{2}{*}{17} & 100 & 2,88 & 1000 & 1,05 \\
& 200 & 1,94 & 2000 & 0,98 \\
& 300 & 1,16 & 3000 & 0,86 \\
\hline \multirow{2}{*}{19} & 100 & 3,74 & 1000 & 1,52 \\
& 200 & 2,39 & 2000 & 0,96 \\
& 300 & 1,43 & 3000 & 0,99 \\
\hline \multirow{2}{*}{21} & 100 & 2,44 & 1000 & 1,06 \\
& 200 & 3,89 & 2000 & 0,94 \\
& 300 & 1,67 & 3000 & 1,09 \\
\hline
\end{tabular}

Fonte: Do autor, 2014.

Tabela 3 - Resultados para redes Neuro-Fuzzy com topologias diferentes

\begin{tabular}{ccc}
\hline Pertinências & $\mathrm{N}^{\mathrm{o}}$ de Épocas & Erro Médio (\%) \\
\hline \multirow{2}{*}{2} & 100 & 7,55 \\
& 200 & 5,91 \\
& 300 & 4,85 \\
\hline \multirow{3}{*}{3} & 100 & 0,72 \\
& 200 & 0,69 \\
& 300 & 0,67 \\
\hline \multirow{2}{*}{4} & 100 & 0,68 \\
& 200 & 0,63 \\
& 300 & 0,55 \\
\hline \multirow{2}{*}{5} & 100 & 0,40 \\
& 200 & 0,12 \\
\hline
\end{tabular}

Fonte: Do autor, 2014. 
Tabela 4 - Resultados para a rede Neuro-Fuzzy com 3 funções de pertinência e diferentes épocas de treinamento

\begin{tabular}{ccc}
\hline Pertinências & $\mathrm{N}^{\mathrm{o}}$ de Épocas & Erro Médio (\%) \\
\hline \multirow{3}{*}{3} & 1000 & 0,45 \\
& 2000 & 0,04 \\
& 3000 & 0,02 \\
\hline
\end{tabular}

Fonte: Do autor, 2014.

Dentre as topologias abordadas neste trabalho, a que apresentou o menor erro relativo médio para o treinamento foi a rede Neuro-Fuzzy com 3 funções de pertinência para cada entrada e número de épocas de treinamento igual a 3000. Considerou-se, portanto, essa rede como a mais adequada para a resolução do problema de medição de energia elétrica. Realizou-se, ainda, a validação dessa rede para comparar seus resultados com os resultados obtidos pela rede de melhor desempenho, desenvolvida pelo artigo base. Essa comparação está descrita pela Tabela 5.

Tabela 5 - Comparativo das validações das redes

\begin{tabular}{cccccc}
\hline $\begin{array}{c}\text { Número do } \\
\text { Ensaio }\end{array}$ & $\begin{array}{c}\text { Valores Reais } \\
(\mathrm{Wh})\end{array}$ & $\begin{array}{c}\text { Valores da Rede de } \\
\text { Referência }(\mathrm{Wh})\end{array}$ & $\begin{array}{c}\text { Erro } \\
(\%)\end{array}$ & $\begin{array}{c}\text { Valores da rede } \\
\text { ANFIS }(\mathrm{Wh})\end{array}$ & $\begin{array}{c}\text { Erro } \\
(\%)\end{array}$ \\
\hline Ensaio 01 & 44,89 & 45,61 & 1,59 & 44,84 & 0,11 \\
Ensaio 02 & 45,77 & 45,54 & 0,51 & 45,49 & 0,61 \\
Ensaio 03 & 741,81 & 726,75 & 2,03 & 747,12 & 0,72 \\
Ensaio 04 & 351,54 & 361,48 & 2,83 & 402,31 & 14,44 \\
\hline
\end{tabular}

Fonte: Do autor, 2014.

Percebe-se que a rede ANFIS proposta neste trabalho apresenta resultados bem próximos dos reais, considerando os Ensaios 01 e 02. Para o Ensaio 03, o desempenho é similar ao da rede proposta pela referência (SOUZA et al., 2010) e, para o Ensaio 04, a rede ANFIS proposta apresenta um erro relativo considerável se comparado com a rede de referência.

\section{CONCLUSÃO}

De modo geral, pode-se dizer que, mesmo com uma quantidade de dados reduzida e levando em consideração a complexidade do tema, as topologias abordadas neste artigo conseguiram mapear, com erro aceitável, o comportamento da medição da energia elétrica frente a distorções harmônicas. 
A topologia Neuro-Fuzzy proposta apresentou resultados próximos dos valores reais. Porém, para o Ensaio 04 em específico, verificou-se um erro relativo elevado podendo caracterizar uma situação de overfitting, um fenômeno que ocorre quando a rede memoriza os dados de entrada e não consegue se adaptae a pequenas variações dos padrões de entrada. Essa afirmação, contudo, só pode ser efetivamente comprovada pela análise de resultados da rede para um conjunto de testes mais representativo. Desse modo será possível, também, dizer se a rede proposta neste artigo consegue ser mais eficiente do que a proposta pela referência (SOUZA et al., 2010).

\section{REFERÊNCIAS}

DUGAN, S. S. R. C.; MCGRANAGHAN, M. F.; BEATY, H. W. Electrical power systems quality. New York: McGraw Hill, 2012.

ELLER, N. A. Arquitetura de informação para o gerenciamento de perdas comerciais de energia elétrica. 2003 Tese (Doutorado em Engenharia de Produção) - Centro Tecnológico, Universidade Federal de Santa Catarina, Florianópolis. 2003. 114 f. apud SOUZA, André Nunes de et al. Inteligência computacional aplicada na identificação e classificação de problemas de medição de energia elétrica. In: BRAZILIAN CONFERENCE ON DYNAMICS CONTROL AND THEIR APPLICATIONS. 9., 2010, Serra Negra. [Anais]... Serra Negra: [s.n.], 2010.

MARRO, A. A. et al. Lógica fuzzy: conceitos e aplicações. Natal: Universidade Federal do Rio Grande do Norte (UFRN), 2010.

RODRIGUES, A. M. Influência da distorção harmônica e de desequilíbrios em medidores de energia elétrica ativa. 2009. 11 p. TCC (Graduação em Engenharia Elétrica) Universidade Federal do Paraná, Curitiba, 2009.

SILVA, I. N. de; SPATTI, D. H.; FLAUZINO, R. A. Redes neurais artificiais para engenharia e ciências aplicadas: curso prático. São Paulo: Artliber, 2010.

SILVA, L. C. E. da. Efeitos das distorções harmônicas (tensões e correntes) e desequilíbrios (tensões) em medidores eletrônicos trifásicos de energia elétrica ativa. 2008. 202 f. Dissertação (Mestrado em Engenharia Elétrica) - Faculdade de Engenharia, Universidade Estadual Paulista, Ilha Solteira, 2008.

SOARES, F. A.; ANDRADE, M. M. de. Apostila de instrumentação e processamento de sinais biológicos. Brasília: [s.n.], 2004.

SOUZA, A. N. de et al. Inteligência computacional aplicada na identificação e classificação de problemas de medição de energia elétrica. In: BRAZILIAN CONFERENCE ON 
DYNAMICS CONTROL AND THEIR APPLICATIONS. 9., 2010, Serra Negra. [Anais]... Serra Negra: [s.n.], 2010.

TEIXEIRA, R. de A.; CORRÊA, M. V.; ASSIS, J. C. de O. Redes neuro-fuzzy: algoritmo de treinamento e aplicações. Principium Online: Iniciação Científica no Unileste-MG, Coronel Fabriciano, v. 1, n. 3, p.152-168, dez. 2007.

Recebido em: 06/06/2015

Aprovado em: 16/06/2015

Publicado em: 29/06/2015 